\title{
BMJ Open Differences between determinants of in-hospital mortality and hospitalisation costs for patients with acute heart failure: a nationwide observational study from Japan
}

Noriko Sasaki, Susumu Kunisawa, Hiroshi Ikai, Yuichi Imanaka

To cite: Sasaki N, Kunisawa S, Ikai $\mathrm{H}$, et al. Differences between determinants of in-hospital mortality and hospitalisation costs for patients with acute heart failure: a nationwide observational study from Japan. BMJ Open 2017;7: e013753. doi:10.1136/ bmjopen-2016-013753

- Prepublication history for this paper is available online. To view these files please visit the journal online (http://dx.doi.org/10.1136/ bmjopen-2016-013753).

Received 4 August 2016 Revised 17 November 2016 Accepted 17 January 2017

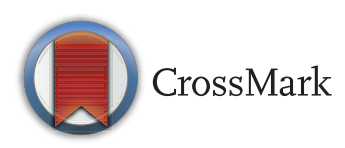

Department of Healthcare Economics and Quality Management, Kyoto University Graduate School of Medicine, Kyoto, Japan

Correspondence to Dr Yuichi Imanaka; imanaka-y@umin.net

\begin{abstract}
Objectives: Although current case-mix classifications in prospective payment systems were developed to estimate patient resource usage, whether these classifications reflect clinical outcomes remains unknown. The efficient management of acute heart failure (AHF) with high mortality is becoming more important in many countries as its prevalence and associated costs are rapidly increasing. Here, we investigate the determinants of in-hospital mortality and hospitalisation costs to clarify the impact of severity factors on these outcomes in patients with AHF, and examine the level of agreement between the predicted values of mortality and costs.
\end{abstract}

Design: Cross-sectional observational study.

Setting and participants: A total of 19926 patients with AHF from 261 acute care hospitals in Japan were analysed using administrative claims data.

Main outcome measures: Multivariable logistic regression analysis and linear regression analysis were performed to examine the determinants of in-hospital mortality and hospitalisation costs, respectively. The independent variables were grouped into patient condition on admission, postadmission procedures indicating disease severity (eg, intra-aortic balloon pumping) and other high-cost procedures (eg, singlephoton emission CT). These groups of independent variables were cumulatively added to the models, and their effects on the models' abilities to predict the respective outcomes were examined. The level of agreement between the quartiles of predicted mortality and predicted costs was analysed using Cohen's $\kappa$ coefficient.

Results: In-hospital mortality was associated with patient's condition on admission and severityindicating procedures (C-statistics 0.870 ), whereas hospitalisation costs were associated with severityindicating procedures and high-cost procedures $\left(R^{2}\right.$ 0.32). There were substantial differences in determinants between the outcomes. In addition, there was no consistent relationship observed $(\kappa=0.016$, $p<0.0001$ ) between the quartiles of in-hospital mortality and hospitalisation costs.

\section{Strengths and limitations of this study}

- The study provides a novel understanding that determinants of in-hospital mortality and hospitalisation costs differ in acute heart failure in Japan.

- Our findings indicate that the same case-mix classifications should not be used to predict both inpatient mortality and costs, which would support the development and implementation of future case-mix classifications.

- External validity of the study could not be determined, and further research is required to investigate the determinants of resources and clinical outcomes in other acute diseases as well as in other settings including other countries.

Conclusions: The determinants of mortality and costs for hospitalised patients with AHF were generally different. Our results indicate that the same case-mix classifications should not be used to predict both these outcomes.

\section{INTRODUCTION}

Prospective payment systems such as the Diagnosis-Related Group (DRG) system in the USA and the Diagnosis Procedure Combination Per-Diem Payment System (DPC/PDPS) in Japan use case-mix classifications that were developed to estimate patient resource usage according to their diseases and underlying conditions. ${ }^{1-6}$ Each DRG or DPC classification includes patients who have similar patterns of resource usage and are comparable from a clinical perspective. The general aim of these systems is to adequately control the inherent variations in patient's disease severity 
and to ensure fair reimbursements to providers that treat different types of patients under limited resources and rising medical costs, especially in the context of increasingly ageing societies. ${ }^{2}$

Despite implicit assumptions that severely ill patients consume more resources, the detailed relationship between severity and outcomes such as mortality and hospitalisation costs remains unknown. ${ }^{7}$ For example, in the All Patients Refined Diagnosis Related Groups (APR-DRG; 3M HIS), ${ }^{1-4} 8$ a patient can be classified into one of the following four levels of severity within each disease category: no/minor complications or comorbidities (CC), moderate CC, major CC, and extreme CG. ${ }^{3}$ These classifications are determined according to each patient's severity of illness and risk of mortality, where the former is used to adjust payment and the latter to adjust mortality rates. ${ }^{1-4} 8$ Similarly, numerous risk-adjustment tools known as severity measures were created and applied in the USA to predict hospital resource consumption and inpatient death in the 1980s and 1990s using multiple payment system data samples. ${ }^{2}{ }^{7-10}$ However, these tools are frequently proprietary, and their underlying logic is unavailable for scrutiny. ${ }^{249}$ The appropriate adjustment of inpatient mortality and hospitalisation costs continues to be an important consideration in different prospective payment systems throughout the world, despite the increasing availability of big data and advances in information technology.

Acute heart failure (AHF) is associated with high hospitalisation and mortality rates, and the rising number of patients with AHF is placing a growing economic burden on healthcare systems; as a result, the efficient management of this disease is being required in various countries. ${ }^{11-13}$ There is an increasing need for the effective and efficient distribution of limited healthcare resources for general healthcare, and also for the treatment of heart failure.

In this study, we aimed to investigate the determinants of in-hospital mortality and hospitalisation costs in patients with AHF to elucidate the impact of patient severity factors, and to examine the level of agreement between the predicted values of mortality and costs.

\section{METHODS}

\section{Data source}

The Quality Indicator/Improvement Project (QIP) is a long-term benchmarking research project involving the voluntary participation of Japanese hospitals that are reimbursed under the DPC/PDPS. ${ }^{5}{ }^{6}$ The DPC/PDPS is a point-based system for hospital reimbursements used by the majority of acute care hospitals in Japan. DPC/ PDPS data are characterised by the inclusion of numerous factors, such as age, sex, admission route, severity levels at admission for specific diseases (eg, New York Heart Association functional class, or NYHA, for AHF; Killip class for acute myocardial infarction; and staging of various cancers), presence of comorbidities, complications, examinations, procedures, medications and costs incurred during hospitalisation.

For this study, data were obtained from 265 acute care hospitals enrolled in the QIP. We analysed patients who had been discharged from these hospitals between April 2010 and March 2011. In order to identify patients with AHF, we first identified patients with a diagnosis of heart failure (International Classification of Diseases, 10th revision code: I50.x) that required the highest usage of healthcare resources for that hospitalisation episode; we then identified patients whose records had an 'acute exacerbation of heart failure' code, which is available in the DPC/PDPS data. Patients were included in the analysis if they were aged 20 years or older on admission and had a length of stay (LOS) duration shorter than 60 days. A total of 25043 patients fulfilled these inclusion criteria. The patient selection process is presented in figure 1 , and the final sample for analysis comprised 19926 patients from 261 hospitals.

\section{Statistical analysis}

The data were analysed using a multivariable logistic regression analysis and linear regression analysis with the dependent variables of in-hospital mortality (dichotomous variable) and hospitalisation costs (continuous variable), respectively. Using these regression models, we examined the factors associated with the target outcome measures. Hospitalisation costs were estimated for each hospitalisation episode using DPC/PDPS points for reimbursements, where each point represents 10 yen. ${ }^{56}$ The predictive abilities of the logistic regression models were assessed using C-statistics, and the predictive abilities of the linear regression models were assessed using $\mathrm{R}^{2}$ values.

The independent variables were categorised into three groups: group 1 involved patient characteristics and condition on admission, and included patient age, sex, route of admission (emergency admission with ambulance use, emergency admission without ambulance use or scheduled admission), NYHA class (II, III or IV) and major comorbidities (hypertension, ischaemic heart disease, atrial fibrillation, fatal arrhythmia and shock). These variables were based on those used in a model that we had previously developed. ${ }^{14}$ Group 2 involved postadmission treatments that may indicate disease severity (hereafter referred to as 'severity-indicating procedures'), and comprised six disease severity classes based on hierarchical and mutually exclusive combinations of the following: percutaneous cardiopulmonary support (PCPS), intra-aortic balloon pumping (IABP), tracheal intubation and catecholamine use. The six classes were PCPS use (regardless of IABP, intubation or catecholamine use), IABP use (regardless of intubation or catecholamine use), intubation or catecholamine use, intubation (with no catecholamine use), catecholamine use only, and none of the aforementioned treatments. In addition, this group included dialysis-related procedures and blood transfusions as independent variables. 
Figure 1 Diagram of patient selection. DPC/PDPS, Diagnosis Procedure Combination Per-Diem Payment System; ICD, International Classification of Diseases; NYHA, New York Heart Association.

Inclusion criteria
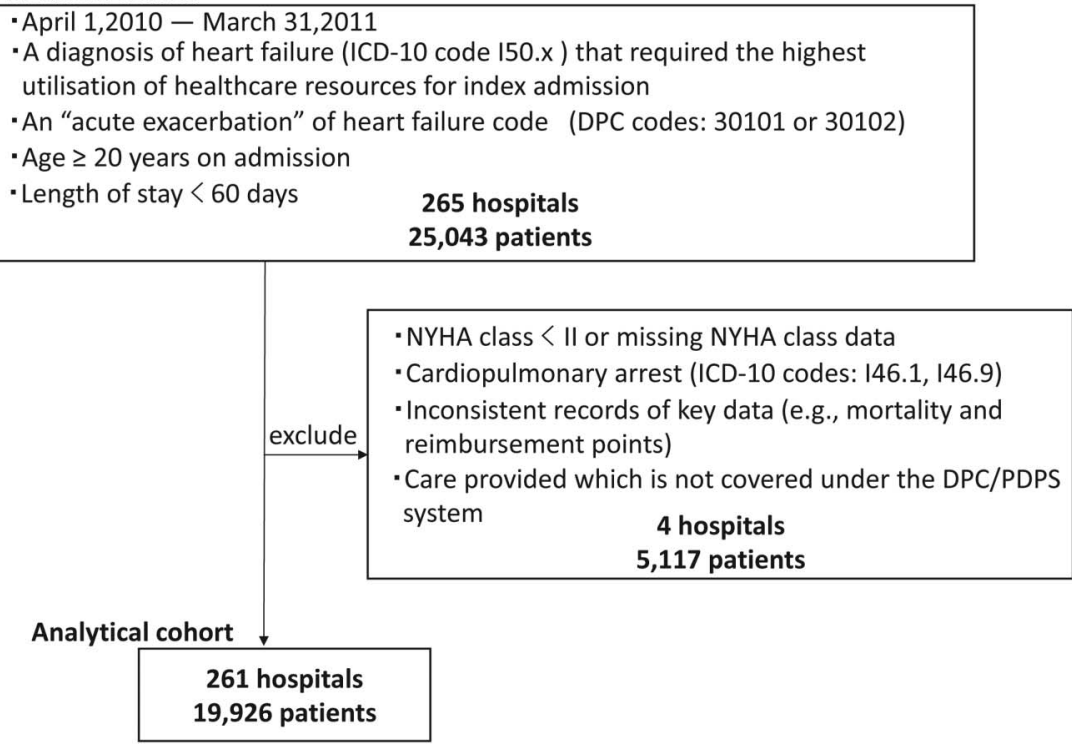

Group 3 involved other postadmission high-cost examinations and treatments performed after admission (hereafter referred to as 'high-cost procedures'), and included percutaneous coronary intervention (PCI), scintigraphy and single-photon emission CT (SPECT).

The three groups of independent variables were sequentially incorporated using the forced entry method into model 1 (group 1 variables only), model 2 (groups 1 and 2 variables only) and model 3 (groups 1-3 variables). The impact of each group of independent variables on the models' predictive abilities was examined. When determining the disease severity class of each patient, we preferentially selected the treatment that indicated the highest level of severity and did not allow for duplicates in cases. As high-cost procedures would have little direct relevance to in-hospital mortality, we did not include these factors as independent variables in the logistic regression models. Therefore, we analysed models 1 and 2 for in-hospital mortality, and models 1-3 for hospitalisation costs.

Next, predicted in-hospital mortality and hospitalisation costs were divided into quartile classes using the estimates from model 2 of the logistic regression model for in-hospital mortality (which included the most clinically relevant independent variables) and model 3 of the linear regression model for hospitalisation costs (which had the highest predictive ability). Cohen's $\kappa$ coefficient was used to evaluate the level of agreement between the two sets of quartile classes to avoid random concordance.

All statistical analyses were conducted using SPSS software V.19.0J (SPSS, Chicago, Illinois, USA). P values (twotailed) below 0.05 were considered statistically significant.

Final cost estimates were converted to US dollars using the 2010 purchasing power parity rate from the Organisation for Economic Co-operation and Development (OECD) data (US $\$ 1.00=111.63$ Japanese yen). ${ }^{15}$

\section{RESULTS}

Hospital and patient characteristics

The 261 acute care hospitals in the study sample had a mean of 353 general care beds (range 30-1106). Among these hospitals, $170(65 \%)$ were accredited as training facilities by the Japanese Circulation Society, and there was a median of 3 cardiologists per facility (range 0-20). The proportions of hospitals according to establishing entity were approximately $18 \%, 35 \%$ and $46 \%$ for public, national and private hospitals, respectively.

The patient characteristics are summarised in table 1 . There was a fairly even distribution between men and women among the 19926 patients. The mean age was 79 years, indicating that the study sample tended towards an older population. Approximately $82 \%$ of the patients were admitted to the emergency department, and $32 \%$ had used ambulances. In addition, $71 \%$ of patients were admitted with moderate-to-high levels of disease severity (NYHA classes III or IV). The unadjusted in-hospital mortality rate was $8.7 \%$, the mean LOS duration was 19.9 days, and the mean hospitalisation cost was US\$8284 per patient.

Moreover, $81 \%$ of the patients did not undergo any severity-indicating procedures, and only $4 \%$ and $7 \%$ of the patients had dialysis-related procedures and blood transfusions, respectively. For other high-cost procedures, $4 \%$ of patients underwent PCI and 9\% underwent SPECT.

\section{Difference in determinants of in-hospital mortality and hospitalisation costs}

The results of the regression analyses to examine the effects of the independent variables on in-hospital mortality and hospitalisation costs are presented in table 2 . In-hospital mortality was analysed using model 1 (group 1 variables only) and model 2 (groups 1 and 2 variables 
Table 1 Patient characteristics, postadmission procedures and patient outcomes

$n=19926$

\begin{tabular}{ll}
\hline Patient characteristics & \\
Women, $\mathrm{n}(\%)$ & $9884(49.6)$ \\
Age in years, mean \pm SD & $79.0 \pm 11.9$ \\
Route of admission, $\mathrm{n}(\%)$ & \\
Emergency with ambulance use & $6393(32.1)$ \\
Emergency without ambulance use & $9903(49.7)$ \\
Scheduled & $3630(18.2)$ \\
New York Heart Association functional class, $\mathrm{n}(\%)$ \\
Class II & $5796(29.1)$ \\
Class III & $7318(36.7)$ \\
Class IV & $6812(34.2)$ \\
Comorbidities, n (\%) & \\
Hypertension & $10588(53.1)$ \\
Ischaemic heart disease & $6120(30.7)$ \\
Atrial fibrillation/flutter & $5369(26.9)$ \\
Fatal arrhythmia & $385(1.9)$ \\
Shock & $321(1.6)$ \\
Postadmision treatments indicating disease sev
\end{tabular}

Postadmission treatments indicating disease severity, n (\%)

Disease severity classes*

No target treatments provided $\quad 16079$ (80.7)

Catecholamine use only $3090(15.5)$

Intubation with no catecholamine use $179(0.9)$

Intubation with catecholamine use $364(1.8)$

Intra-aortic balloon pumpingt 194 (1.0)

Percutaneous cardiopulmonary $20(0.1)$

supportł

Dialysis-related procedures $\quad 790(4.0)$

Blood transfusion 1340 (6.7)

Other postadmission high-cost examinations and

treatments, $\mathrm{n}(\%)$

Percutaneous coronary intervention

$840(4.2)$

Scintigraphy

$226(1.1)$

Single-photon emission CT

$1840(9.2)$

Patient outcomes

In-hospital mortality, $\mathrm{n}(\%) \quad 1725$ (8.7)

Length of stay, days (mean; SD; $\quad 19.9 ; 12.1$;

median)

17.0

Hospitalisation costs (US\$) (mean; SD; 8284; 7448;

median)

6473

${ }^{*}$ These six disease-severity classes are based on hierarchical and mutually exclusive combinations.

tRegardless of intubation or catecholamine use.

$\ddagger$ Regardless of intra-aortic balloon pumping, intubation or catecholamine use.

only), whereas hospitalisation costs were also analysed using model 3 (groups 1-3 variables).

In the logistic regression analysis for in-hospital mortality, the C-statistics (95\% CI) for models 1 and 2 were 0.805 (0.794 to 0.815$)$ and 0.870 (0.862 to 0.879$)$, respectively. Although the inclusion of patient characteristics and condition on admission imparted a fairly high level of predictive ability on in-hospital mortality, the addition of severity-indicating procedures further improved predictive ability. Model 2 identified the following postadmission treatments to be significantly associated with increased in-hospital mortality: catecholamine use only (OR 5.54), intubation (OR 12.70), intubation or catecholamine use (OR 20.97), and PCPS use (OR 42.05). The OR for IABP was relatively lower at 8.06. In addition, dialysis-related procedures (OR 1.73) and blood transfusions (OR 1.38) were also associated with higher in-hospital mortality, although the OR was noticeably lower than that of the disease severity classes.

In the linear regression analysis for hospitalisation costs, the $\mathrm{R}^{2}$ values for models $1-3$ were $4 \%, 18 \%$ and $32 \%$, respectively. Model 1 , which included only patient characteristics and condition on admission, had a low coefficient of determination. However, the inclusion of severity-indicating procedures in model 2 resulted in an approximately fourfold increase in predictive ability, and the further inclusion of high-cost procedures in model 3 increased predictive ability almost eightfold. Model 3 identified the following determinants of hospitalisation costs: emergency admission with ambulance use (increase of approximately US $\$ 520$ ), NYHA class III or IV on admission (US\$490 and US\$640, respectively), fatal arrhythmia (US\$4550), ischaemic heart disease (US $\$ 410$ ) and atrial fibrillation (US\$460). All factors in the severity-indicating procedures and high-cost procedures were also significantly associated with higher hospitalisation costs. In particular, the following had a substantial impact on increasing costs: IABP and PCI use (both associated with an increase of US\$13 850); PCPS use (US\$8360); blood transfusion, scintigraphy or SPECT (US\$3600); and dialysis-related procedures (US\$2120).

NYHA class and severity-indicating procedures were associated with both increased in-hospital mortality and hospitalisation costs. In contrast, several patient characteristics (age, route of admission and comorbidities) demonstrated conflicting effects on the outcomes. For example, age appeared to be strongly associated with increased mortality but lower costs, whereas ischaemic heart disease was associated with lower mortality but higher costs. Route of admission and fatal arrhythmia were significantly associated with higher hospitalisation costs, but had no effect on in-hospital mortality.

Figure 2 shows the association between the unstandardised coefficients of in-hospital mortality risk (model 2) and hospitalisation costs (model 3). Although increased age was generally associated with higher mortality but lower costs, extremely ill patients who required PCPS were associated with higher mortality and lower costs when compared with similar patients who required only IABP.

\section{Poor agreement between the quartile classes of predicted} in-hospital mortality and hospitalisation costs

Table 3 shows the $4 \times 4$ contingency table of the quartile classes of predicted in-hospital mortality (model 2) and predicted hospitalisation costs (model 3). Agreement between the quartile classes of these model 2 outcomes was extremely poor $(\kappa=0.016, p<0.0001)$, indicating that predicted mortality had no association with predicted hospitalisation costs. 
Table 2 Determinants of in-hospital mortality and hospitalisation costs

\begin{tabular}{|c|c|c|c|c|c|}
\hline \multirow{3}{*}{$\begin{array}{l}\text { Dependent variables } \\
\text { Independent variables }\end{array}$} & \multicolumn{2}{|c|}{ In-hospital mortality } & \multicolumn{3}{|c|}{ Hospitalisation costs } \\
\hline & Model 1 & Model 2 & Model 1 & Model 2 & Model 3 \\
\hline & \multicolumn{2}{|l|}{ OR } & \multicolumn{3}{|c|}{ Unstandardised coefficients (US\$) } \\
\hline \multicolumn{6}{|l|}{ Patient characteristics } \\
\hline Women (reference category: men) & 0.923 & 1.017 & -205 & -109 & 36 \\
\hline \multicolumn{6}{|l|}{ Age (reference category: $20-59$ years) } \\
\hline $60-69$ years & 1.334 & 1.307 & 313 & 222 & 250 \\
\hline $70-79$ years & $2.257^{\star \star \star}$ & $2.661^{\star \star \star}$ & -189 & -60 & 18 \\
\hline $80-89$ years & $4.146^{\star \star \star}$ & $6.009^{\star \star \star}$ & $-1181^{\star \star \star}$ & $-827^{\star \star \star}$ & $-454^{\star}$ \\
\hline$\geq 90$ years & $7.086^{\star \star \star}$ & $12.751^{\star * *}$ & $-2027^{\star \star \star}$ & $-1400^{\star * *}$ & $-678^{\star \star}$ \\
\hline \multicolumn{6}{|c|}{ Route of admission (reference category: scheduled) } \\
\hline Emergency with ambulance use & $1.185^{\star}$ & 0.891 & $1201^{\star \star \star}$ & $772^{\star \star \star}$ & $521^{\star \star \star}$ \\
\hline Emergency without ambulance use & 0.916 & $0.807^{\star}$ & $530^{\star \star \star}$ & $422^{* *}$ & $276^{\star}$ \\
\hline \multicolumn{6}{|c|}{ NYHA functional class (reference category: class II) } \\
\hline Class III & $2.121^{\star \star \star}$ & $2.011^{\star \star \star}$ & $608^{\star \star \star}$ & $467^{\star \star \star}$ & $494^{\star \star \star}$ \\
\hline Class IV & $6.812^{\star \star \star}$ & $5.929^{\star \star \star}$ & $1121^{\star \star \star}$ & $727^{\star \star \star}$ & $635^{\star \star \star}$ \\
\hline \multicolumn{6}{|l|}{ Comorbidities } \\
\hline Hypertension & $0.307^{\star \star *}$ & $0.32^{\star \star *}$ & -200 & 61 & -120 \\
\hline Ischaemic heart disease & $0.573^{\star * *}$ & $0.522^{\star \star \star}$ & $1520^{\star \star \star}$ & $1484^{\star * *}$ & $409^{\star \star *}$ \\
\hline Atrial fibrillation/flutter & $0.561^{\star \star \star}$ & $0.604^{\star \star \star}$ & -145 & $333^{\star \star}$ & $457^{\star \star \star}$ \\
\hline Fatal arrhythmia & $1.637^{\star \star}$ & 1.081 & $5635^{\star \star \star}$ & $4597^{\star \star *}$ & $4547^{\star \star \star}$ \\
\hline Shock & $3.058^{\star * \star}$ & 1.189 & $3335^{\star \star \star}$ & -584 & -412 \\
\hline \multicolumn{6}{|c|}{ Postadmission treatments indicating disease severity } \\
\hline \multicolumn{6}{|c|}{ Disease severity classes† (reference category: no target treatments provided) } \\
\hline Catecholamine use only & - & $5.543^{\star \star \star}$ & - & $1740^{\star \star *}$ & $1565^{\star \star \star}$ \\
\hline Intubation with no catecholamine use & - & $12.699^{\star \star \star}$ & - & $1253^{*}$ & $1162^{\star}$ \\
\hline Intubation or catecholamine use & - & $20.966^{\star \star \star}$ & - & $1681^{\star \star *}$ & $1610^{\star \star \star}$ \\
\hline Intra-aortic balloon pumpingł & - & $8.062^{\star \star *}$ & - & $22628^{\star * *}$ & $13854^{\star * *}$ \\
\hline Percutaneous cardiopulmonary support§ & - & $42.048^{\star \star \star}$ & - & $15421^{\star \star \star}$ & $8360^{\star \star \star}$ \\
\hline Dialysis-related procedures & - & $1.729^{\star \star \star}$ & - & $2739^{\star \star \star}$ & $2117^{\star \star \star}$ \\
\hline Blood transfusion & - & $1.383^{\star \star}$ & & $4186^{\star * *}$ & $3884^{\star \star \star}$ \\
\hline \multicolumn{6}{|c|}{ Other postadmission high-cost examinations and treatments } \\
\hline Percutaneous coronary intervention & - & - & - & - & $13842^{\star \star \star}$ \\
\hline Scintigraphy & - & - & - & - & $3683^{\star \star \star}$ \\
\hline Single-photon emission CT & - & - & - & - & $3324^{\star \star \star}$ \\
\hline C-statistics (95\% Cls) & $\begin{array}{l}0.805 \\
(0.794-0.815)\end{array}$ & $\begin{array}{l}0.870 \\
(0.862-0.879)\end{array}$ & - & - & - \\
\hline $\mathrm{R}^{2}$ & - & - & 0.044 & 0.178 & 0.320 \\
\hline
\end{tabular}

Model 1: independent variables included only patient characteristics and condition on admission; model 2: independent variables included postadmission treatments that may indicate disease severity in addition to those of model 1; model 3: independent variables included other high-cost examinations and treatments in addition to those of model 2.

${ }^{* * *} p<0.001 ;{ }^{* *} p<0.01 ;{ }^{*} p<0.05$.

†These six disease-severity classes are based on hierarchical and mutually exclusive combinations.

†Regardless of intubation or catecholamine use.

$\S$ Regardless of intra-aortic balloon pumping, intubation or catecholamine use.

NYHA, New York Heart Association.

\section{DISCUSSION}

In the present study, we conducted a detailed examination of the determinants of in-hospital mortality and hospitalisation costs in a variety of AHF practice settings. In particular, our analysis found exceedingly poor agreement between the quartile classes of in-hospital mortality and hospitalisation costs, which indicated a lack of a consistent relationship between the outcomes.

In table 1 , the majority of patients with AHF (approximately $80 \%$ ) did not undergo any of the target severity-indicating procedures, which is consistent with analyses of global registries in several countries. ${ }^{12}$ In consideration of this severely ill portion of the population, there is a need to focus on accurately identifying the cost drivers and accounting for the appropriate predictors of hospitalisation costs that are distinct from the predictors of mortality. Therefore, we discriminated severity-indicating procedures which have a greater impact on mortality than on hospitalisation costs and other high-cost procedures which are more important predictors of hospitalisation costs in this study.

The combination of patient condition on admission, severity-indicating procedures and high-cost procedures had differing levels of influence on predicting mortality 


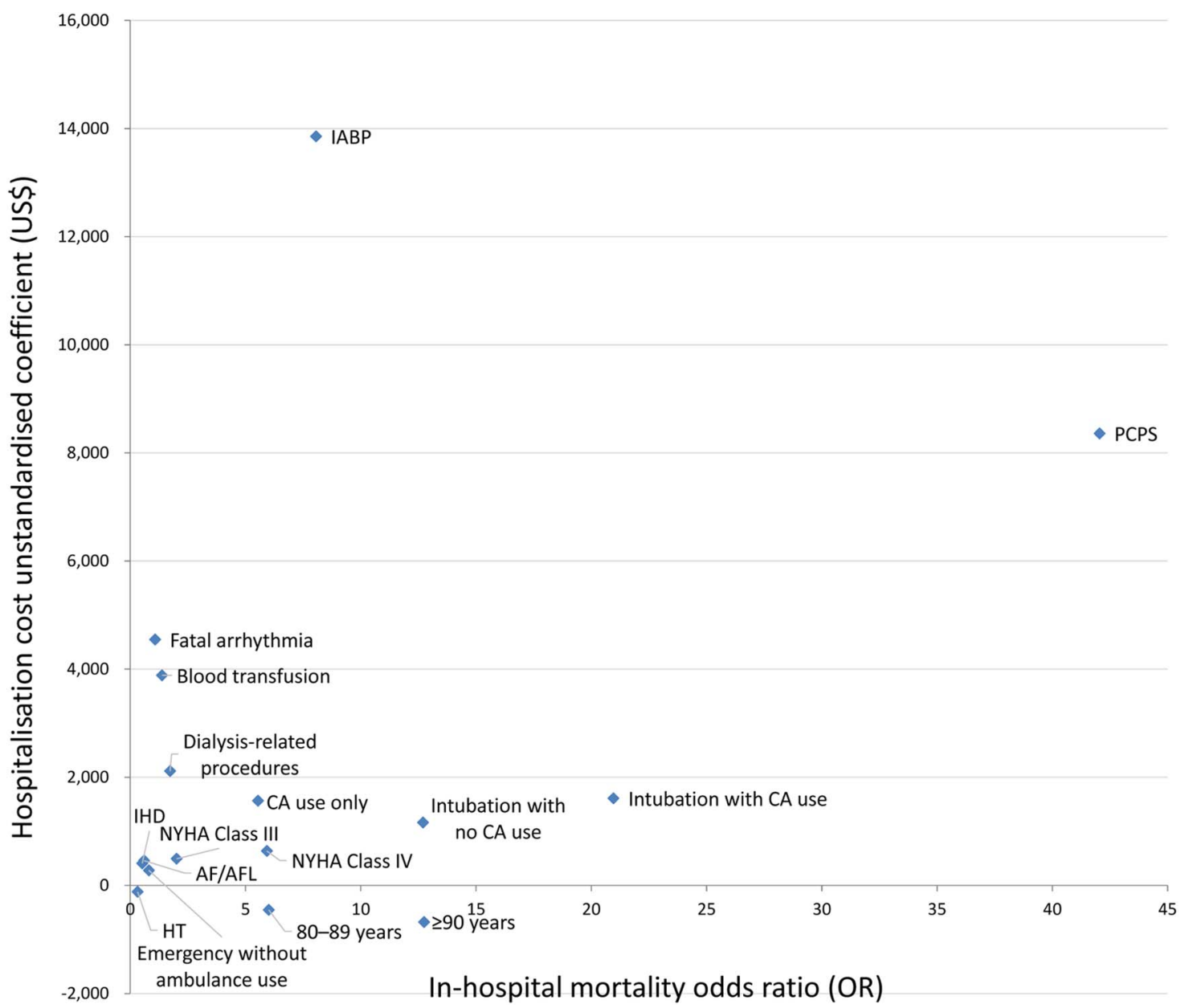

Figure 2 Relationship between the in-hospital mortality (model 2) and the hospitalisation costs (model 3). AF/AFL, atrial fibrillation/atrial flutter; CA, catecholamine; HT, hypertension; IABP, intra-aortic balloon pumping; IHD, ischaemic heart disease; NYHA class, New York Heart Association functional class; PCPS, percutaneous cardiopulmonary support.

Table 3 Poor agreement between the quartile classes of predicted in-hospital mortality and hospitalisation costs

\begin{tabular}{|c|c|c|c|c|c|c|}
\hline \multirow[b]{2}{*}{$n(\%)$} & & \multicolumn{4}{|c|}{ Predicted hospitalisation costs* } & \multirow[b]{2}{*}{ Total } \\
\hline & & $\begin{array}{l}\text { Low } \longleftarrow \\
\text { Class } 1\end{array}$ & Class 2 & Class 3 & $\begin{array}{l}\text { high } \\
\text { Class } 4\end{array}$ & \\
\hline \multicolumn{7}{|c|}{ Predicted in-hospital mortality* } \\
\hline \multirow{3}{*}{$\begin{array}{c}\text { Low } \\
\uparrow\end{array}$} & Class 1 & $1243(6.2)$ & $1415(7.1)$ & $1209(6.1)$ & $1014(5.1)$ & $4881(24.5)$ \\
\hline & Class 2 & $1672(8.4)$ & $1250(6.3)$ & $1054(5.3)$ & $1104(5.5)$ & $5080(25.5)$ \\
\hline & Class 3 & $1380(6.9)$ & $1394(7.0)$ & $1036(5.2)$ & 1174 (5.9) & $4984(25.0)$ \\
\hline \multirow[t]{2}{*}{ High } & Class 4 & $692(3.5)$ & $908(4.6)$ & $1689(8.5)$ & $1692(8.5)$ & $4981(25.0)$ \\
\hline & Total & $4987(25.0)$ & 4967 (24.9) & $4988(25.0)$ & $4984(25.0)$ & $19926(100.0)$ \\
\hline
\end{tabular}

Numbers in parentheses indicate percentages of the total; Cohen's $\kappa=0.016, p<0.0001$.

Class 1: below the lower quartile; class 2: between the lower quartile and median; class 3: between median and upper quartile; class 4: above upper quartile.

*Model 2 of in-hospital mortality and model 3 of hospitalisation costs were used for prediction.

and costs. Specifically, model 2 (involving patient condition on admission and severity-indicating procedures) had a high level of predictive ability for mortality (C-statistic 0.870). For hospitalisation costs, patient condition on admission alone provided a negligible degree of predictive ability $\left(R^{2} 0.044\right)$. However, the determinant of coefficient rose considerably with the inclusion of severity-indicating procedures in model $2\left(\mathrm{R}^{2} 0.178\right)$, and rose further with the inclusion of high-cost procedures in model $3\left(R^{2} 0.320\right.$; table 2$)$. The extremely poor agreement level between the quartile classes of mortality and costs clarified the lack of a consistent relationship between the outcomes, which was beyond our expectations (table 3 ). 
Interestingly, our study further revealed that the implicit assumption that severely ill patients will consume more resources is not necessarily true, especially when a patient is extremely ill and close to death; this was similar to the findings of a previous study. ${ }^{7}{ }^{16}$ Figure 2 demonstrates that an association between older age and lower hospitalisation costs, which is consistent with an earlier report. ${ }^{13}$ Moreover, IABP had a relatively lower risk for mortality (OR 8.06) than intubation (OR 12.70), intubation or catecholamine use (OR 20.97), and PCPS use (OR 42.05); however, IABP was associated with the highest additional costs but lowest mortality among them. In contrast, patients who required PCPS demonstrated the highest risk of mortality but lower costs than IABP patients. Continuing life-prolonging care provided in IABP patients was considered to be a major contributing factor of increasing costs compared with PCPS patients who generally die soon. We could learn the differential impact of IABP and PCPS on the two outcomes as a representative example which seemed to be clinically plausible.

The implications of our findings are that the same case-mix classifications should not be used to predict both costs and mortality in patients with AHF. Disease-specific case-mix classifications in consolidated DRG or DPC groups would not accurately predict mortality. A previous study has reported that mortality rates for individual DRGs are a poor measure of quality, even under an optimistic assumption of perfect case-mix adjustment. ${ }^{17}$ In addition, our study demonstrated that the segregation of specific high-cost procedures that indicate disease severity and other high-cost procedures such as PCI, scintigraphy and SPECT may help to explain inpatient mortality and costs more accurately.

To the best of our knowledge, this is the first study to investigate both the determinants and inter-relationship between in-hospital mortality and hospitalisation costs in AHF participants across Japan. Previous studies from other countries have reported conflicting results in the relationship between costs and mortality, ${ }^{16}{ }^{18-20}$ and some have reported that decreased resource usage leads to worse outcomes. ${ }^{20}$ This study found that the determinants of mortality and costs for hospitalised patients with AHF were generally different. Accordingly, these findings would support the accurate assessment of resources and quality, which is an important step in the future development and implementation of case-mix classifications.

This study had the following limitations. First, the sample hospitals were limited to those that have adopted the DPC/PDPS. Moreover, the inherent differences in healthcare systems among countries may limit the generalisability of our findings. However, this study focused on patients with AHF, and our sample included a wide variety of acute care hospitals. As a result, our analysis may be fairly robust to variations in patient and hospital characteristics. Second, it is difficult to directly compare mortality (a dichotomous variable) and hospitalisation costs (a continuous variable). However, we were able to clarify the differential impact of various disease severity classes and other independent variables available in the clinical setting to each of the outcome measures, and used the $\kappa$ coefficient to demonstrate the poor agreement between the outcomes. Third, the individual expertise and experience of each physician may induce variations in the provision of high-cost procedures, ${ }^{6}$ but we were unable to investigate these factors using administrative data. Fourth, the absence of ventricular function data may decrease our ability to assess severity of illness at admission. Finally, the external validity of the study could not be determined, and further research is required to investigate the determinants of outcomes in other acute diseases as well as in other settings including other countries.

\section{CONCLUSIONS}

The determinants of in-hospital mortality and hospitalisation costs were generally different in patients with AHF. Our results indicate that the same case-mix classifications should not be used to predict both costs and mortality.

Contributors NS and $\mathrm{YI}$ had full access to all the data, were involved in the study design and wrote the manuscript. NS, SK, HI and YI acquired, analysed and interpreted the data, as well as performed the statistical analyses. YI acquired funding for the study. All authors were involved in critical revisions and approval of the final manuscript.

Funding This research was financially supported in part by a Health Sciences Research Grant from the Ministry of Health, Labour and Welfare of Japan (H27-iryo-ippan-001), a Grant-in-Aid for Scientific Research from the Japan Society for the Promotion of Science ([A]16H02634), and a Health, Labour and Welfare Policy Research Grant (H28-seisaku-shitei-009).

Competing interests None declared.

Ethics approval This study was approved by the Ethics Committee of Kyoto University Graduate School and Faculty of Medicine, Japan.

Provenance and peer review Not commissioned; externally peer reviewed.

Data sharing statement No additional data are available.

Open Access This is an Open Access article distributed in accordance with the Creative Commons Attribution Non Commercial (CC BY-NC 4.0) license, which permits others to distribute, remix, adapt, build upon this work noncommercially, and license their derivative works on different terms, provided the original work is properly cited and the use is non-commercial. See: http:// creativecommons.org/licenses/by-nc/4.0/

\section{REFERENCES}

1. Cretin S, Worthman LG. Alternative systems for case-mix classification in health care financing. R-3457-HCFA. Prepared for the Health Care Financing Administration, U.S Department of Health and Human Services. The RAND Publication Series. ISBN 0-83300763-7 http://www.acove.com/content/dam/rand/pubs/reports/2008/ R3457.pdf

2. lezzoni L, ed. Risk adjustment for measuring health care outcomes. 4th edn. Chicago: Health Administration Press, 2003.

3. Rutledge R, Osler T. The ICD-9-based illness severity score: a new model that outperforms both DRG and APR-DRG as predictors of survival and resource utilization. J Trauma 1998;45:791-9.

4. Kongstvedt PR, Goldfield NI, Plocher DW. Using data and provider profiling in medical management. In: Kongstvedt PR, ed. Essentials of managed healthcare. 4th edn. MA: Jones and Bartlett Publishers, 2003: 379-418.

5. Anderson G, Ikegami N. How can Japan's DPC inpatient hospital payment system be strengthened? Lessons from the U.S. Medicare 
prospective system. A report of the CSIS global health policy center. 2011.

6. Hamada H, Sekimoto M, Imanaka Y. Effects of the per diem prospective payment system with DRG-like grouping system (DPC/ PDPS) on resource usage and healthcare quality in Japan. Health Policy 2012;107:194-201.

7. McMahon LF Jr, Newbold RR. Variation in resource use within diagnosis-related groups. The effect of severity of illness and physician practice. Med Care 1986;24:388-97.

8. Romano PS, Chan BK. Risk-adjusting acute myocardial infarction mortality: are APR-DRGs the right tool? Health Serv Res 2000;34:1469-89.

9. lezzoni LI, Ash AS, Shwartz M et al. Predicting who dies depends on how severity is measured: implications for evaluating patient outcomes. Ann Intern Med 1995;123:763-70.

10. McMahon LF Jr, Billi JE. Measurement of severity of illness and the Medicare prospective payment system: state of the art and future directions. J Gen Intern Med 1988;3:482-90.

11. Lloyd-Jones D, Adams RJ, Brown TM, et al., Writing Group Members. Heart disease and stroke statistics-2010 update: a report from the American Heart Association. Circulation 2010;121: e46-e215.

12. Ambrosy AP, Fonarow GC, Butler $\mathrm{J}$ et al. The global health and economic burden of hospitalizations for heart failure: lessons learned from hospitalized heart failure registries. J Am Coll Cardiol 2014;63:1123-33.

13. Smith DH, Johnson ES, Blough DK et al. Predicting costs of care in heart failure patients. BMC Health Serv Res 2012;12:434-42.

14. Sasaki N, Lee J, Park S, et al. Development and validation of an acute heart failure-specific mortality predictive model based on administrative data. Can J Cardiol 2013;29:1055-61.

15. OECD Data. Purchasing power parities (PPP). Total, National currency units/US dollar, 2000-2015. https://data.oecd.org/conversion/ purchasing-power-parities-ppp.htm (accessed 17 Nov 2016).

16. Freeman JL, Fetter RB, Park H et al. Diagnosis-Related Group refinement with diagnosis- and procedure-specific comorbidities and complications. Med Care 1995;33:806-27.

17. Hofer TP, Hayward RA. Identifying poor-quality hospitals: can hospital mortality rates detect quality problems for medical diagnoses? Med Care 1996;34:737-53.

18. Kruse M, Christensen J. Is quality costly? Patient and hospital cost drivers in vascular surgery. Health Econ Rev 2013;3:22-35.

19. Schreyogg J, Stargardt T. The trade-off between costs and outcomes: the case of acute myocardial infarction. Health Serv Res 2010;45:1585-601.

20. Stargardt T, Schreyogg J, Kondofersky I. Measuring the relationship between costs and outcomes: the examples of AMI in German hospitals. Health Econ 2014;23:653-69. 